\title{
Hybrid Quantum Nanophotonic Devices for Coupling to Rare-Earth Ions
}

\author{
Evan Miyazono, Alex Hartz, Tian Zhong, Andrei Faraon \\ T. J. Watson Laboratory of Applied Physics, California Institute of Technology, 1200 East California Boulevard, \\ Pasadena, CA 91125, USA \\ miyazono@caltech.edu
}

\begin{abstract}
Gallium arsenide photonic crystal resonators are designed and fabricated for evanescent coupling to localized ensembles of rare-earth ions in crystalline hosts. These devices will enable nano-scale on-chip optical quantum memories.
\end{abstract}

(C)2014 Optical Society of America

OCIS codes: (270.0270) Quantum optics, (230.0230) Optical devices, (270.5580) Quantum electrodynamics, (160.5690) Rare-earth-doped materials.

Developing quantum nanophotonic devices coupled to rare-earth ions (REIs) in crystalline hosts will enable new systems that could act as on-chip light matter interfaces. With the narrowest optical line-widths, very long spin coherence times and strong dipole-dipole interactions, the rare-earth ions are some of the most fascinating light emitters embedded in solids [1]. However, the weak oscillator strength and the lack of fabrication techniques for their crystalline hosts made it challenging so far to develop nanophotonic devices based on these light emitters.

The strength of the REI optical transition can be strongly enhanced via the Purcell effect by coupling to an optical nano-cavity with large quality factor and small mode volume [2]. The number of atoms required to significantly affect the cavity field is proportional to the cavity loss and inversely proportional to the coupling rate between the atom and the cavity field [3]. Minimizing this number requires a small mode volume and a large quality factor. Using optical nano-resonators with optical mode volumes $(\mathrm{V})$ of a few cubic wavelengths and quality factors on the order of $\mathrm{Q}=10^{5}$, the transition is enhanced such that even a single rare-earth ion can affect the transmission function of the resonator as shown in Fig. 1, for a $\mathrm{Nd}$ ion embedded in yttrium orthosilicate (YSO).

(a)

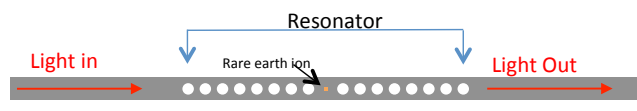

(b)

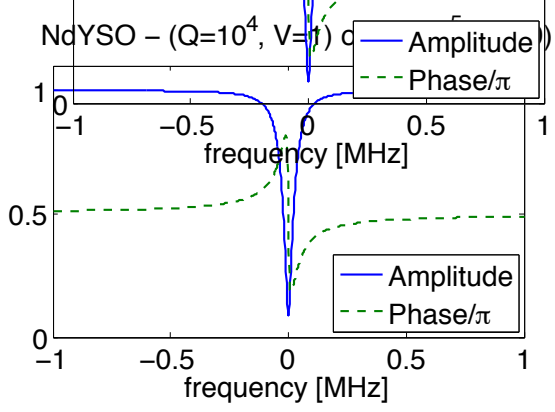

Fig. 1: (a) Schematic representation of a REI coupled to photonic crystal nano-beam resonator formed by etching nano-scale holes in a beam of high-index material with sub-micron width and height. (b) Calculated transmission spectrum of the Nd ion coupled to the nano-beam. By scanning a laser through the cavity resonance, the signature of only one single ion can be detected as a sharp change in the transmission function. The parameters for the resonator, $\mathrm{Q}=10^{4}, \mathrm{~V}=1$ for the nano-beam or $\mathrm{Q}=10^{5}, \mathrm{~V}=10$ for the ring, are routinely achievable in many material systems.

To develop these devices, we use a hybrid method where a high-index semiconductor, gallium arsenide (GaAs), resonator sits on the rare-earth-doped crystal (YSO) and the REI $\left(\mathrm{Nd}^{3+}\right)$ dopants couple to the evanescent cavity field, represented in Fig. 2(a). The $\mathrm{Nd}^{3+}$ ions have optical transitions at $883 \mathrm{~nm}$. GaAs membranes (164 nm thick) grown epitaxially on top of an AlGaAs layer on a GaAs wafer are used in this experiment. We optimized designs for nanobeam photonic crystal cavities with quality factors of 288,000 and mode volumes of 0.58 cubic wavelengths, which will enable coupling with high cooperativity to $\mathrm{Nd}$ ions. The field profile for the resonator (optimized using finite difference time-domain simulations) is shown in Fig. 2(b). In Fig. 2(c), we show that the electric field at the interface between GaAs and YSO is only a factor of 0.6 lower than the field maximum in the resonator thus enabling efficient coupling to the rare-earth ions via the evanescent field. We also investigate microdisk and microring 
photonic crystal cavities where quality factors exceeding $10^{5}$ can be achieved while mode volumes remain only a few cubic wavelengths.

We developed two fabrication procedures for the hybrid devices. In one procedure the devices are first fabricated on the GaAs wafer using electron-beam lithography and dry plasma etching. Then the AlGaAs layer is etched using a drop of hydrofluoric acid (HF) and the devices are released in the HF solution. The solution is then placed on top of the YSO chip and a significant fraction of the devices become bonded on the YSO substrate after the solution evaporates. In Fig. 2(d) and Fig. 3(c) we show devices fabricated using this procedure. In another procedure we wafer bond the GaAs membrane on the YSO chip before fabricating the resonators.
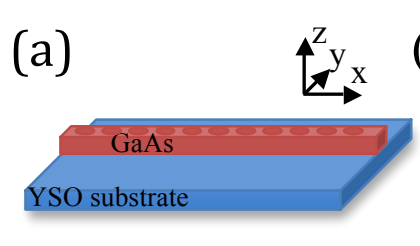

(b)

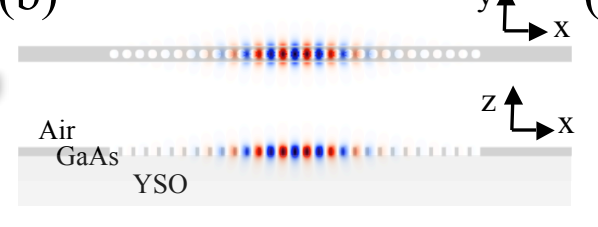

(c)

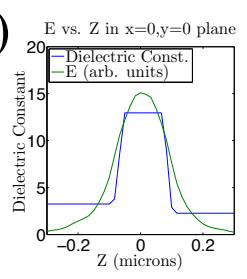

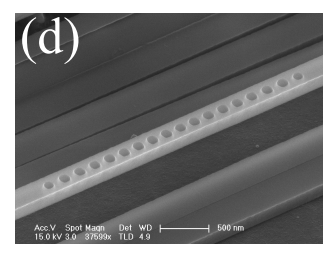

Fig. 2: (a) Concept of the hybrid photonic platform where the resonator is fabricated in a high-index material located on top of the YSO crystal. (b) Simulation of the Ey electric field in a GaAs nanobeam photonic crystal cavity. (c) The magnitude of the electric field along the z-axis in the middle of the cavity. (d) Fabricated GaAs nano-beam photonic crystal cavities with AlGaAs sacrificial layer locally etched, suspending the structures for testing before transferring to a YSO chip.

The devices are measured in a confocal microscope setup. The light is coupled into the nano-beam resonators using a grating coupler. The input beam from a supercontinuum source is focused onto one grating coupler while light transmitted through the structure is scattered out of and gathered from the other grating coupler, as illustrated in Fig. 3(a). In a simple beam expander, a pinhole is used to spatially filter the collected light, transmitting only light from the output grating. This light is sent to a spectrometer, and an example spectrum can be seen in Fig. 3(b). In this case we show the resonances of the microdisk resonator, and fitting Lorentzian distributions allows us to calculate the quality factor of these resonances to be $\mathrm{Q} \sim 5000$.
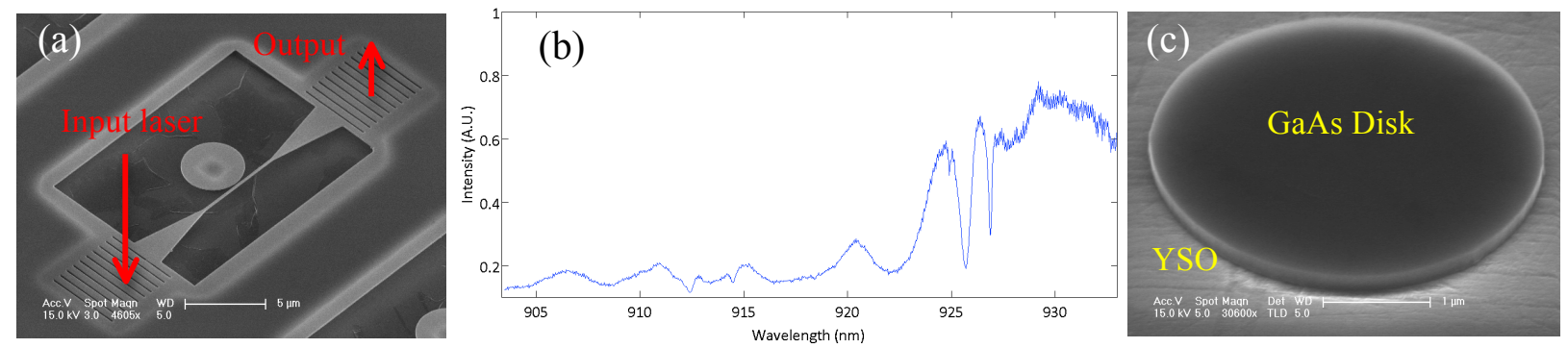

Fig. 3: Device measurement. (a) Scanning electron microscope image of a waveguide-coupled GaAs microdisk resonator illustrating spatial separation of input and output grating couplers (b) Measured transmission through the disk-coupled waveguide, demonstrating a resonance with quality factor $\mathrm{Q} \sim 5000$ at $926.8 \mathrm{~nm}$. (c) Scanning electron microscope image of a GaAs microdisk resonator transferred onto a YSO substrate.

In conclusion, we demonstrate nanophotonic devices for coupling to Nd ions in YSO. These devices are expected to enable optical coupling with high cooperativity at the level of single photons and single ion and will be used as efficient on-chip light-matter interfaces.

\section{References}

[1] D. L. McAuslan, J. J. Longdell, R. J. Sellars, "Strong-coupling cavity QED using rare-earth-metal-ion dopants in monolithic resonators: What you can do with a weak oscillator," Phys. Rev. A 80, 062307 (2009).

[2] A. Faraon, P. E. Barclay, C. Santori, K. C. Fu and R. G. Beausoleil, "Resonant enhancement of the zero-phonon emission from a colour centre in a diamond cavity," Nature Photon. 5, 301-305 (2011).

[3] D. L. McAuslan, D. Korystov, and J. J. Longdell, "Coherent spectroscopy of rare-earth-metal-ion-doped whispering-gallery-mode resonators," Phys. Rev. A 83, 063847 (2011). 